\title{
MATRIZ DE COMPETENCIAS DEL DOCENTE DE EDUCACIÓN BÁSICA
}

\author{
José M. Fernández \\ Universidad Politécnica Antonio José de Sucre
}

\section{INTRODUCCIÓN}

En las dos últimas décadas ha irrumpido con fuerza en las áreas de Gestión de los Recursos Humanos y de la Educación una nueva palabra clave: Competencia. Los propósitos de muchos programas educativos ya no están definidos en función de objetivos sino de competencias. Las características que definen un cargo se describen por competencias. A las personas se les evalúa para indagar si tienen suficiencia para optar a un título o un puesto de trabajo por las competencias que demuestran. Competencia es un término globalizado. Se utiliza lo mismo en Australia que en Canadá, en México que en Colombia o Venezuela. En muchos países del mundo se ha creado un sistema de cualificación laboral y de formación por competencias. El Programa de Educación básica de $1^{0}$ a $6^{\text {to }}$ grado vigente en Venezuela tiene por finalidad desarrollar en los niños competencias fundamentales para su desarrollo posterior.

\section{PLANTEAMIENTO DEL PROBLEMA}

Los interrogantes que se plantean en esta investigación son los siguientes: ¿Cuáles son los significados de competencia? ¿Qué elementos esenciales contiene? ¿De dónde viene su origen? ¿Qué usos tiene este concepto? ¿Tiene aplicación el concepto de competencia a la profesión del docente? ¿Cómo se identifican las competencias del docente de Educación básica? ¿Cuáles son las competencias del docente de Educación básica? ¿Qué aplicaciones tiene la definición de una mat riz de competencias del docente de Educación básica?.

\section{OBJETIVO GENERAL}

El objetivo del presente trabajo de investigación consiste en precisar los elementos esenciales del concepto de competencia, y ofrecer una matriz de las competencias subcompetencias e indicadores del docente de Educación básica, elaborada por los mismos docentes mediante una metodología de Focus Group.

\section{OBJETIVOS ESPECÍFICOS}

1.- Presentar las diferentes visiones, evolución y conceptos de Competencia.

2.- Identificar los elementos esenciales de una competencia..

3.- Planificar y ejecutar una metodología para elaborar el perfil de competencias del Docente de Educación básica.

4.- $\quad$ Producir una matriz de competencias del docente de Educación básica.

\section{JUSTIFICACIÓN}

El objetivo que se propuso la presente investigación fue la de elaborar un perfil o una matriz de competencias del docente de Educación Básica. La inquietud por realizar el estudio surgió a raíz de la 
lectura de las 10 competencias del docente propuestas por Perrenoud que son las siguientes: (1) Organizar y dirigir situaciones de aprendizaje, (2) Administrar la progresión de los aprendizajes, (3) Concebir y hacer evolucionar los dispositivos de diferenciación, (4) Envolver a los alumnos en sus aprendizajes y en su trabajo, (5) Trabajar en equipo, (6) Participar en la administración de la escuela, (7) Informar y envolver a los pares, (8) Utilizar nuevas tecnologías, (9) Enfrentar los deberes y los dilemas éticos de la profesión, (10) Administrar su propia formación continua.

Estos atributos del docente se formularon en otro tiempo y otro contexto. ¿Porqué no indagar cuál es el perfil de competencias del maestro de básica en Venezuela?. ¿Porqué no son los mismos docentes quienes descubran su propio perfil de competencias?.

Dos razones importantes justifican este esfuerzo: (1) Los programas de 1ro a 6to grado (lamentablemente la reforma educativa sólo llegó hasta 6to grado) de educación en Venezuela tienen como propósito desarrollar las competencias en los alumnos, las mismas están formuladas por asignatura y grado, ¿Porqué no identificar pues cuál es el perfil de competencias que debe mostrar el maestro?. (2) Es cierto que en Venezuela los subsistemas de ingreso a la docencia, remuneración y evaluación se rigen por reglamentos y contratos cuyos mandatos no guardan relación con una gestión de recursos humanos por competencias.

¿Pero cómo programar la capacitación de los docentes en ejercicio sin establecer previamente el nivel óptimo de desempeño de los mismos, con base en las competencias que deben cualificarlos en función de un mejoramiento continuo de la calidad educativa?

\section{ORIGEN, USOS Y CONCEPTO DE COMPETENCIA}

El vocablo competencia es polisémico. El diccionario de la Real Academia Española le atribuye varios significados diferentes unos de otros. Por una parte competencia significa "disputa o contienda entre dos o más personas sobre algo; Situación de empresas que rivalizan en el mercado ofreciendo o demandando un mismo producto o servicio; Competencia deportiva". Por otra parte le atribuye estos significados: 1.- Incumbencia; 2.- Pericia, aptitud, idoneidad para hacer algo o intervenir en un asunto determinado; 3. - Atribución legítima a un juez u otra autoridad para el conocimiento o resolución de un asunto".

Se puede entender competencia como: (1) Rivalidad deportiva o empresaria: Competencia deportiva, Competencia empresarial, (2) Asignación de responsabilidad a una institución sobre determinadas materias: Competencias administrativas, regionales o a determinadas audiencias judiciales, (3) Pericia, aptitud o idoneidad para hacer algo. Este informe se referirá a esclarecer los elementos contenidos o incorporados por los psicólogos, investigadores de Recursos Humanos y Educadores a la tercera acepción del vocablo.

Hacia 1970, las empresas y organizaciones seleccionaban el personal para incorporarlo a su organización, mediante test de inteligencia y exámenes de conocimiento. Creían que las personas con mayor coeficiente intelectual y con mejores notas podían ser los profesionales más exitosos en las organizaciones. Mc Clelland (citado en HayGroup, 1996) profesor de la Universidad de Harvard inició una investigación sobre el porqué unos empleados tienen más éxito que otros en el trabajo. Por medio del método de incidentes críticos identificó qué cualidades o aptitudes hacían que un trabajador lograra 
resultados excepcionales. Dicho estudio le llevó a la conclusión que no había correlación entre los profesionales de mayor coeficiente intelectual y el éxito sino que este dependía de otras características personales como aptitudes y motivaciones.

Daniel Goleman (1999) popularizó el concepto de Inteligencia Emocional, contraponiendo ésta a la Inteligencia Racional, definiéndola como "la capacidad de reconocer nuestros propios sentimientos y los ajenos, de motivarnos y de manejar bien nuestras emociones", en el mismo texto cita una definición más precisa de Salovey y Mayer: "en función de la capacidad de monitorear y regular los sentimientos propios y ajenos, y de utilizar los sentimientos para guiar el pensamiento y la acción". Lo interesante en los estudios de Goleman es que investigando las características para 181 puestos en 121 compañías internacionales y diferenciando las aptitudes meramente cognitivas o técnicas de las emocionales, descubrió que las dos terceras partes del éxito de los trabajadores se debía a las aptitudes emocionales y la otra tercera parte a las intelectuales.

La empresa asesora internacional de recursos humanos Hay/McBer (citado en HayGroup, 1996) llegó con sus investigaciones a resultados parecidos. Es decir, el éxito de un trabajador, de un profesional o una persona se debe en mayor medida a sus características personales no intelectuales ni meramente técnicas. Esta organización promocionaba un modelo de gestión de Recursos Humanos fundamentado en la definición y descripción de un puesto de trabajo, caracterizado éste por la delimitación de un área de responsabilidad del puesto dentro de una estructura organizativa. Este puesto se definía por funciones y los que ocuparan los cargos debían tener determinado nivel profesional y demostrar algunas habilidades cognitivas o técnicas. Este modelo correspondía a la concepción de empresa fordista donde lo importante era la estructura y la organización.

Pero las investigaciones realizadas por la misma empresa y la revolución ocurrida en las organizaciones con la irrupción de la sociedad del conocimiento llevaron a la misma a proponer otro modelo de gestión de Recursos Humanos. Las Organizaciones deben ser flexibles, adaptarse a los cambios, trabajar en equipos, preocuparse por estándares y resultados, ser competitivas. Lo importante ya no son los cargos sino las competencias de quienes integran la empresa.

Boyatzis (citado en HayGroup, 1996) define la competencia como "una característica subyacente en una persona que está causalmente relacionada con una actuación exitosa en un puesto de trabajo,... Las competencias pueden consistir en motivos, rasgos de carácter, conceptos de uno mismo, actitudes o valores". Cualquier característica individual que se pueda medir de un modo fiable y que se pueda demostrar que diferencia al trabajador que tiene un desempeño con resultados excelentes del que no lo tiene.

Toda competencia contiene tres elementos básicos: Características personales, Ejecuciones y Resultados positivos. La Gestión integrada de Recursos Humanos gira en torno a este concepto de Competencia. Una vez definidas las competencias requeridas para un cargo, se selecciona el personal, se evalúa el desempeño, se valora el potencial, se programa la capacitación y el plan de carrera de las personas.

Debido a la crisis del modelo fordista, a los cambios acelerados ocurridos en el sector productivo, a los procesos de reconversión industrial y al desempleo, los gobiernos conjuntamente con los sindicatos y los empresarios, sintieron la necesidad de conjugar esfuerzos y programas con el sector educativo para 
responder al reto que suponía la sociedad del conocimiento. En casi todos los países desarrollados primero, y luego en los países en vías de desarrollo se fueron imponiendo Sistemas Nacionales de Cualificación y Formación Laboral, fundamentando estos en el modelo de Competencias.

Es así como Inglaterra en la década de los 80 crea el Consejo Nacional para las Calificaciones Profesionales que irán definiendo y certificando el sistema de Calificaciones Profesionales Nacionales. Sistemas parecidos se crean en los diferentes países de la Comunidad Europea, en Estados Unidos, Canadá y Australia. En la década de los 90 nace en México el Sistema Normalizado de Competencia Laboral y su complemento natural La Educación Basada en Normas de Competencia. Varios países latinoamericanos están instrumentando sistemas parecidos basados en competencias.

Hasta ahora se ha analizado el término de competencia desde la perspectiva del trabajo y la productividad. ¿Pero cómo se percibe este concepto desde el punto de vista educativo? ¿Está en concordancia su significado con las corrientes pedagógicas actuales? ¿Qué variables educativas pueden enriquecer el término competencia?. Una guía excepcional para indagar la evolución filosófica, pedagógica, y política de la Educación en el mundo nos la brinda la UNESCO, a través de sus documentos e informes. Dos de estos informes son realmente esclarecedores ya que recogen todas las corrientes filosóficas, pedagógicas y políticas de los países del mundo y proponen líneas de acción que profundicen la esencia del hecho educativo para todos los habitantes del planeta.

El primer informe titulado Aprender a Ser publicado en la década de los 70, o también llamado Informe Faure por el nombre del presidente de la comisión internacional que redactó el documento, marca ya una diferencia con respecto a la línea tradicional de la Educación. Tradicionalmente el hecho educativo ponía su acento en la instrucción y en la concepción de que el alumno era sujeto pasivo de la recepción de conocimientos que poseía el profesor que era quien administraba la educación. El Informe Aprender a Ser pone el acento no tanto en el educar sino en el aprender y por tanto convierte al estudiante en sujeto activo de su propio desarrollo.

El alumno es el centro del hecho educativo. El alumno debe aprender a ser persona, ciudadano, sujeto de derechos y deberes. Este informe incorpora como eje de la política educativa el concepto de Educación Permanente en el que todas las personas son sujetos para aprender durante toda la vida y pueden decidir qué, cómo y cuándo aprender.

El segundo se titula La Educación Encierra un Tesoro y fue publicado en 1996. Es importante resaltar dos aspectos fundamentales del documento que tienen que ver con el tema que nos ocupa. El primero habla de la filosofía de la Educación, entendiendo que ésta no es meramente un medio, un capital humano para el crecimiento económico, sino que el aprendizaje es esencial al desarrollo humano y por tanto es un fin en sí mismo. "La Educación debe facilitar a todos, lo antes posible el pasaporte para la vida, que le permitirá comprenderse mejor a sí mismo, entender a los demás y participar así en la obra colectiva y la vida en sociedad" (Delors 1996). El segundo aspecto se refiere a los principios de la Educación:

La educación a lo largo de la vida se basa en cuatro pilares: Aprender a conocer, aprender a hacer, aprender a vivir juntos, aprender a ser. Aprender a conocer, combinando una cultura general suficientemente amplia con la posibilidad de profundizar los conocimientos en un pequeño número de materias. Aprender a hacer a fin de adquirir no solo una calificación profesional, sino más generalmente, una competencia que capacite al individuo para hacer frente a gran número de situaciones y a trabajar en equipo. Aprender a vivir juntos desarrollando la comprensión del otro y la percepción de las formas de interdependencia respetando los valores del pluralismo, 
comprensión mutua y paz. Aprender a ser para que florezca mejor la propia personalidad y se esté en condiciones de obrar con creciente capacidad de autonomía, de juicio y de responsabilidad personal. Mientras los sistemas educativos formales propenden a dar prioridad a la adquisición de conocimientos, en detrimento de otras formas de aprendizaje, importa concebir la educación como un todo. En esta concepción deben buscar inspiración y orientación las reformas educativas, tanto en la elaboración de los programas como en la definición de las nuevas políticas pedagógicas. (Delors, 1996).

En este texto se establecen algunos lineamientos fundamentales: (1) El Aprendizaje no es sólo cognitivo sino desarrollo de aptitudes, capacidades, competencias para hacer, ser y convivir; (2) Estas cuatro dimensiones del aprendizaje deben considerarse como un todo. No están aisladas en el hecho educativo sino que conforman una totalidad. Deben globalizarse los aprendizajes; (3) Estos aprendizajes están en función de una vida más plena, con más posibilidades y libertad, para la convivencia y para producir en equipo, para disfrutar del sentido estético, de las capacidades físicas, de lo espiritual; (4) El ser, el conocer, el hacer y el convivir se aprenden o se desarrollan y deben orientar las reformas educativas y los programas de estudio.

Desde la perspectiva de la filosofía, Sicología y Pedagogía también han cambiado las teorías del aprendizaje. Durante la mayor parte del siglo XX, los modelos educativos estuvieron sustentados por las teorías del Conductismo. Según esta teoría, el objeto de la Sicología y de la Pedagogía es el estudio y cambio de las conductas humanas entendidas estas como manifestaciones observables y medibles, externas a la persona que responden a determinados estímulos y gratificaciones.

Para el Conductismo, la persona es como una caja negra. No interesa su interior puesto que no se puede observar. Lo que sí se puede acreditar son los estímulos que recibe la persona y las respuestas que manifiesta. Los programas educativos estuvieron diseñados con base en objetivos generales y específicos a lograr, casi siempre cognitivos. Para superar estos objetivos era imprescindible aprobar exámenes o pruebas objetivas si se deseaba ascender un peldaño más en la carrera educativa o profesional. Los estudios estaban condicionados por perfiles y programas externos al individuo. Este se convertía en un sujeto pasivo que debía responder a las expectativas del profesor si quería ser gratificado con su aprobación.

En la segunda mitad del siglo XX se desarrolló y propagó la teoría del constructivismo que sirve de soporte para muchos programas educativos actuales. Según este modelo, el aprendizaje lo va construyendo el individuo en interacción permanente y continua con los objetos de conocimiento, en intercambios sociales y según la importancia que asigne a los aprendizajes. El individuo es una persona que tiene intereses y conceptos previos y a través de ellos interactúa con las percepciones externas y con los entornos sociales para ir generando nuevos conceptos, visiones, aptitudes, motivaciones y formas de actuar. Las características internas del aprendiz, sus mapas mentales, el significado que asigne al mundo externo que se le presente, sus motivaciones, participan activamente en la formación de nuevos aprendizajes cognitivos, procedimentales o actitudinales. En tal sentido hay correspondencia entre la teoría constructivista y el concepto de competencia.

De lo visto anteriormente se deduce que, con diferentes matices, tanto el modelo de Gestión de Recursos Humanos como el educativo y la teoría constructivista convergen en el concepto de competencia. El concepto de competencia es el nuevo paradigma de la Educación y de la gestión de Recursos Humanos. de tal forma que se convierte en bisagra, en moneda de cambio, entre el sector laboral y el formativo. 
Independientemente de que este concepto está demasiado circunscrito al ámbito laboral y de formación para el mismo, su significado más profundo recoge la globalidad del principio fundamental que propone la UNESCO sobre los cuatro pilares de la Educación. Es decir, las competencias recogen en una totalidad, conocimientos, atributos personales, convivencia y ejecuciones que logran resultados, así estos sean de tolerancia, democracia, paz, satisfacción y plenitud humanas.

Se han dado múltiples definiciones de competencia tanto de especialistas en la materia como de instituciones de cualificación y formación laboral. Vargas Zúñiga (2004) recoge en su libro "Cuarenta preguntas sobre competencia laboral", doce definiciones de distintos especialistas y veinte de Instituciones Nacionales o de Formación a nivel mundial. Unas acentúan los atributos o características personales de quienes las poseen, otras el desempeño en función de resultados exitosos. Otros señalan la capacidad de lograr resultados en situaciones diferentes y adversas. Conviene citar aquí cinco de estas definiciones, tres de especialistas y dos de Instituciones:

Una compleja estructura de atributos necesarios para el desempeño en situaciones específicas (Gronzci)...Capacidad real para lograr un objetivo o resultado en un contexto dado (Mertens)... Una construcción a partir de una combinación de recursos (conocimientos, saber hacer, cualidades o aptitudes) y recursos del ambiente (relaciones, documentos, informaciones y otros) que son movilizados para lograr un desempeño (Le Boterf)... Capacidad de articular, movilizar y colocar en acción, valores, conocimientos, y habilidades necesarias para el desempeño eficiente y eficaz de actividades requeridas por la naturaleza del trabajo (Ministerio de Educación de Brasil)... Es un conjunto de comportamientos socioafectivos y habilidades cognoscitivas sicológicas sensoriales y motoras que permiten llevar a cabo adecuadamente un papel, una función una actividad o una tarea (Provincia de Québec).

De todas ellas se pueden deducir los elementos esenciales: (1) Son características o atributos personales: conocimientos, habilidades, aptitudes, rasgos de carácter, conceptos de uno mismo; (2) Están causalmente relacionados con ejecuciones que producen resultados exitosos. Se manifiestan en la acción; (3) Son características subyacentes a la persona que funcionan como un sistema interactivo y globalizador, como un todo inseparable que es superior y diferente a la suma de atributos individuales; (4) Logran resultados en diferentes contextos. Estos cuatro elementos son esenciales al concepto de competencia y no puede darse la misma si falta uno de éstos.

\section{METODOLOGÍA}

¿Cómo se construyó el perfil de competencias del docente de Educación Básica que se presentará en los resultados?. Se utilizó el método más comúnmente utilizado para definir competencias. Son los mismos profesionales quienes deben identificar aquellas competencias que conforman la esencia de los cargos que ocupan para que estos sean exitosos. En el caso que nos ocupa se seleccionaron dos grupos de aproximadamente 20 docentes de Educación Básica. motivados por el auto aprendizaje. El primer grupo lo conformaban maestros de aula de 5 escuelas estadales del Municipio Autónomo Caroní. En el segundo grupo participaron docentes adscritos al Ministerio de Educación del mismo Municipio.

La forma de abordar el tema fue a través de la técnica del focus goup, tal como lo entiende Korman (citado en la página Web de la Universidad de Antioquia, 2004): "una reunión de un grupo de individuos seleccionados por los investigadores para discutir y elaborar desde la experiencia personal una temática o hecho social que es objeto de investigación". Los grupos focales requieren de procesos de interacción, discusión y elaboración de unos acuerdos dentro del grupo acerca de unas temáticas que son propuestas por los investigadores. 
¿En qué consistió el proceso de elaboración de la matriz de competencias?. En la primera sesión se explicaron los objetivos del taller, se presentaron los conceptos básicos de la administración de Recursos Humanos por competencias y se presentó el modelo de competencia utilizado por la empresa HayGroup (1996) tal como aparece en el grafico 1.

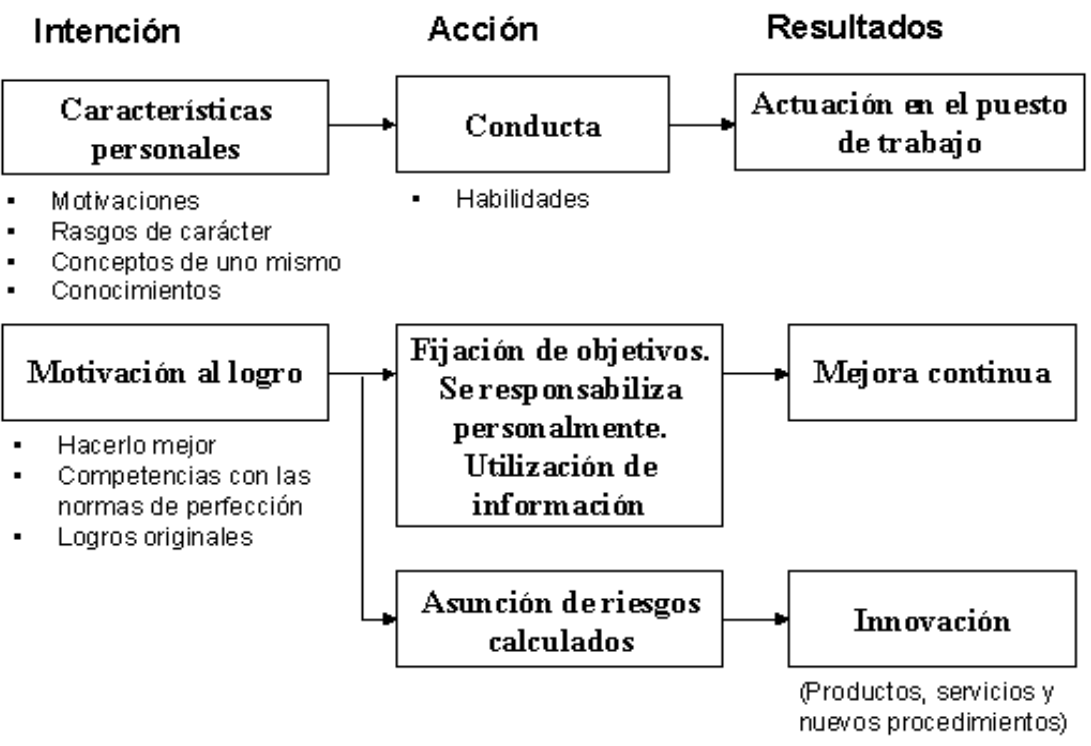

Grafico 1. Modelo de Flujo Causal de Competencia. Fuente: HayGroup, 1996.

Una vez establecido el consenso en cuanto a la definición de conceptos a utilizar, se presentaron e interpretaron los objetivos de la Escuela Básica definidos por los emisarios de todo el mundo en la Conferencia de Jontiem (UNESCO, 2000) tal como aparecen en el gráfico 2. Venezuela es firmante de estos acuerdos.

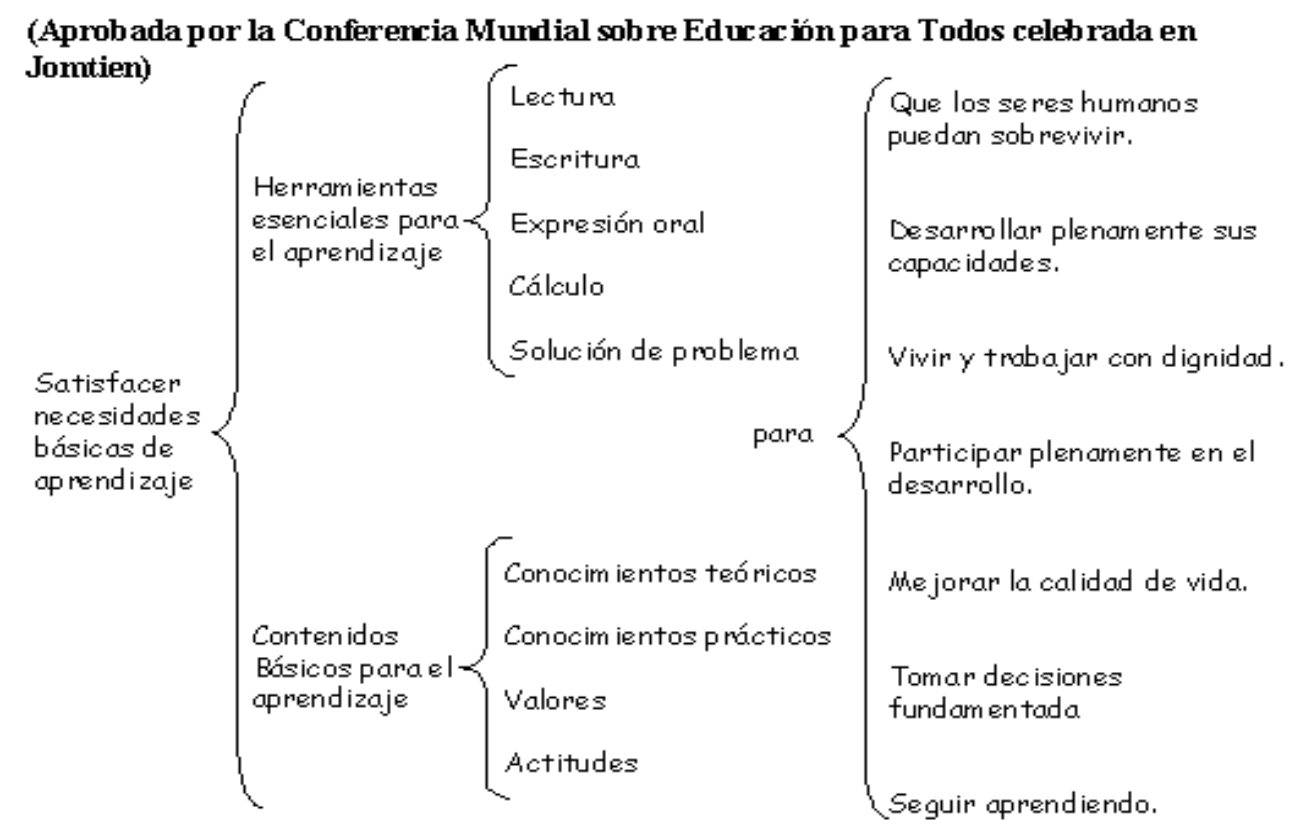

Grafico 2. Declaración Mundial sobre Educación para Todos. Fuente: UNESCO, 2000.

Al final de la discusión hubo consenso para aceptar que esos eran los resultados que debe lograr un maestro en todos sus alumnos de acuerdo a su nivel y grado. Hay que insistir mucho en este aspecto ya que en general no existe conciencia en los profesores de que ellos son exitosos si logran resultados en los 
alumnos. A continuación se le pidió a cada uno de los participantes que pensara en un maestro que él consideraba que lograba en los alumnos los resultados antes presentados y que listara en una hoja todas las actuaciones que ejecutaba para lograr tales objetivos.

Se insistió mucho en que fueran ejecuciones y no conceptos generales que en concreto no identifican la causa de los resultados. Al final de la reunión en pequeños grupos se sumaron las ejecuciones, se eliminaron las que se repetían y cada grupo trató de agrupar las acciones en conjuntos que según ellos respondían a algunos atributos personales. Se instó a los docentes a desechar conceptos vagos e imprecisos que se repiten como una retahíla.

Entre la primera y la segunda sesión se transcribieron los listados de todos los maestros tal como ellos lo habían escrito. Este fue el insumo básico para la segunda sesión y estos son los indicadores que aparecen en la matriz. En esta reunión se volvieron a recordar los conceptos básicos de la primera y en base al modelo de competencias a seguir. Se partió de los resultados exitosos para identificar las acciones que causaban tales resultados. En esta sesión se debían agrupar todas las acciones señaladas por afinidad en 10 conjuntos diferentes. Cada conjunto de indicadores debía llevar al grupo a identificar alguna característica personal del docente de la cual procedían tales acciones.

Dicha sesión produjo como resultado una primera identificación de diez competencias con sus indicadores. Para la siguiente sesión, los docentes de cada escuela participante debían presentar dos competencias con subcompetencias e indicadores completando, ordenando y adecuando el material previamente elaborado. Entre la tercera y la cuarta sesión se presentaron las competencias, se discutieron y consensuaron los nombres de las competencias y subcompetencias y de esta forma se logró la matriz de Competencias del docente de Educación Básica que se muestra en los resultados.

\section{RESULTADOS}

La matriz de competencias resultante se presenta de la siguiente manera. Los números naturales del 1 al 10 representan las competencias, cada una se divide en subcompetencias y para cada subcompetencia se señalan algunos indicadores de ejecución.

1. Motivación al logro.

1.1. Espíritu de superación y logro de metas.

$\checkmark \quad$ Fija metas, planifica y evalúa a corto, mediano y largo plazo.

$\checkmark \quad$ Establece prioridades.

$\checkmark \quad$ Organiza recursos en función de resultados. Diagnostica, programa, ejecuta y evalúa.

$\checkmark \quad$ Se evalúa en forma continua para reorientar y cambiar de estrategias.

1.2. Espíritu de trabajo e innovación.

$\checkmark \quad$ Manifiesta interés por las actividades ejecutadas en la institución.

$\checkmark \quad$ Planifica proyectos innovadores.

$\checkmark \quad$ Planifica y organiza día a día su actividad pedagógica.

$\checkmark \quad$ Refuerza las competencias difíciles de lograr.

2. Atención centrada en el alumno.

2.1 Empatía con el alumno.

Es amigo de los alumnos. 
$\checkmark \quad$ Paciente y tolerante con el proceso de aprendizaje del alumno.

$\checkmark \quad$ Se preocupa y motiva a los niños. Flexible con los alumnos. Orienta e los niños.

2.2 Diagnostica al grupo de alumnos.

$\checkmark \quad$ Elabora el perfil de entrada de los alumnos del grado.

$\checkmark \quad$ Parte del conocimiento previo de los alumnos.

$\checkmark \quad$ Observa fortalezas y debilidades de los alumnos.

$\checkmark \quad$ Intercambia ideas con los alumnos.

$\checkmark \quad$ Redacta informes diagnósticos.

2.3 Planifica ejecuta y evalúa actividades dirigidas al pensamiento lógico y creativo del alumno.

$\checkmark \quad$ Maneja los conceptos básicos de Sicología y etapas de aprendizaje de los niños.

$\checkmark \quad$ Promueve la aplicación de procesos: observación, descripción, seriación, clasificación, comparación.

$\checkmark \quad$ Promueve el desarrollo del pensamiento lógico y creativo del alumno.

$\checkmark \quad$ Aplica la resolución de problemas como herramienta para el aprendizaje.

$\checkmark \quad$ Conjuga el uso de estrategias de aprendizaje: memoria, elaboración y aplicación.

$\checkmark \quad$ Adecua el conocimiento al nivel de los niños.

3. Sensibilidad social.

3.1 Conocimiento del entorno.

$\checkmark \quad$ Conocer las características del niño, sus dificultades, aspiraciones, su entorno socialeconómico, sus condiciones de vida, etc.

$\checkmark \quad$ Demuestra interés, respeto y confianza hacia sus representantes, propiciando un acercamiento permanente.

$\checkmark \quad$ Establece reglas claras de convivencia familiar.

3.2 Trabajo en equipo (docente-alumno- representantes- escuela- comunidad).

$\checkmark \quad$ Identidad con la comunidad.

$\checkmark \quad$ Participa y colabora en la solución de problemas de escuela - comunidad.

$\checkmark \quad$ Establece relaciones que permitan integrarse a todos, de tal forma que el problema sea de todos y no de uno.

$\checkmark \quad$ Involucra a los representantes a ser parte de la solución de los problemas de su escuela.

4. Agente de cambio.

\subsection{Motivador}

$\checkmark \quad$ Tiene un compromiso para quienes la escuela es su mejor posibilidad de acceso al conocimiento.

$\checkmark \quad$ Utiliza estrategias novedosas (creativo).

$\checkmark \quad$ Crea un clima participativo.

$\checkmark \quad$ Estimula y promueve la participación de todos.

4.2 Actitud de cambio.

$\checkmark \quad$ Disposición a trabajar en condiciones adversas para el logro del fin ético de la educación.

$\checkmark \quad$ Propone hacer de la escuela una comunidad que aprende.

$\checkmark \quad$ Capacidad para instrumentar cambios.

$\checkmark \quad$ Utilización adecuada del recurso.

$\checkmark \quad$ Reflexión permanente sobre su práctica profesional. 
$\checkmark \quad$ Apertura al cambio y la flexibilidad, para enfrentar la incertidumbre.

5. Equipo de aprendizaje.

5.1 Interdependencia positiva.

$\checkmark \quad$ Compartir recursos.

$\checkmark \quad$ Se ayudan entre si para aprender.

$\checkmark \quad$ Garantizan con su responsabilidad individual el trabajo del grupo.

$\checkmark \quad$ Enseña sus propios conocimientos a los compañeros.

5.2 Habilidades interpersonales y pequeños grupos.

$\checkmark \quad$ Tiene liderazgo.

$\checkmark \quad$ Toma decisiones oportunas.

$\checkmark \quad$ Crea un clima de confianza y comunicación.

$\checkmark \quad$ Maneja conflictos.

5.3 Procesamiento grupal.

$\checkmark \quad$ Discute sobre el logro de objetivos.

$\checkmark \quad$ Evalúa las relaciones de trabajo.

$\checkmark \quad$ Evalúa constantemente las acciones positivas y negativas de los miembros del grupo.

6. Dominio cognoscitivo de los contenidos programáticos de Educación Básica.

6.1 Conocimientos lingüísticos.

$\checkmark \quad$ Domina la competencia comunicativa básica (escuchar-hablar, leer-escribir).

$\checkmark \quad$ Conoce y usa adecuadamente la macroestructura y macroproposiciones textuales.

$\checkmark \quad$ Usa las normas lingüísticas, fonéticas, sintácticas y gramaticales de la lengua española.

6.2 Conocimiento lógico-matemático.

$\checkmark \quad$ Domina con propiedad las operaciones aritméticas.

$\checkmark \quad$ Conoce los principios fundamentales del álgebra, la geometría y la estadística.

$\checkmark \quad$ Redacta y resuelve problemas relacionados a las distintas disciplinas matemáticas.

6.3 Conocimientos de las ciencias experimentales.

$\checkmark \quad$ Conoce los fundamentos y metodología del método científico.

$\checkmark \quad$ Tiene conocimiento de los principios básicos de física y química.

$\checkmark \quad$ Domina los aspectos fundamentales de ciencias biológicas.

6.4 Conocimiento de las ciencias sociales.

$\checkmark \quad$ Conoce los aspectos fundamentales de la historia universal, nacional y local.

$\checkmark \quad$ Domina los principios de la sociología.

$\checkmark \quad$ Conoce los aspectos fundamentales de la geografía.

6.5 Conocimiento de expresión plástica.

$\checkmark \quad$ Domina los principios de expresión plástica.

$\checkmark \quad$ Conoce técnicas relacionadas a las distintas expresiones artísticas.

$\checkmark \quad$ Conoce aspectos fundamentales de la cultura tradicional.

7. Dominio de herramientas de enseñanza y aprendizaje.

7.1 Domina las estrategias para el desarrollo de habilidades cognitivas.

$\checkmark \quad$ Diseña estrategias de aprendizaje.

$\checkmark \quad$ Propicia la divergencia, análisis y producción de ideas para resolver problemas.

$\checkmark \quad$ Se atreve a darle rienda suelta a la creatividad e iniciativa. 
$\checkmark \quad$ Discute planteamientos temáticos que se involucre en el entorno.

$\checkmark \quad$ Planteamiento de situaciones problemáticas a fin de buscar una solución.

7.2 Utiliza herramientas de aprendizaje para la lectura, el calculo, la ciencia y tecnología, y la identidad nacional y local.

$\checkmark$ Aborda lectura y calculo a través de poemas, leyendas, dramatizaciones, adivinanzas, canciones y ejercicios de material concreto.

$\checkmark \quad$ Aplica estrategias de evaluación formativa.

$\checkmark \quad$ Resuelve ejercicios de matemáticas a través de formulación de situaciones de la vida diaria.

$\checkmark \quad$ Aborda la lectura, escritura y calculo con estrategias dinámicas de la realidad del niño con participación directa (manipulando, ejecutando y revisando su trabajo).

$\checkmark \quad$ Relaciona el conjunto de acciones docentes con los acontecimientos de contexto local, regional y nacional.

7.3 Maneja estrategias de motivación.

$\checkmark \quad$ Evalúa de forma permanente su acción aplicando instrumentos o técnicas que involucren a todos los actores de su acción educativa.

$\checkmark \quad$ Tiene destrezas y habilidades de conducción de grupos.

$\checkmark \quad$ Se atreve a darle rienda suelta a la creatividad e iniciativa.

$\checkmark \quad$ Hace uso de actividades de motivación: lluvia de ideas, completación de frases, etc.

$\checkmark \quad$ Reconoce los logros de los alumnos de forma verbal en el grupo.

8. Crea un ambiente de aprendizaje adecuado.

8.1 Ambiente físico y de recursos.

$\checkmark \quad$ Organiza el lugar para propiciar el intercambio de ideas.

$\checkmark \quad$ Acondiciona el espacio físico con materiales informativos apropiados.

$\checkmark \quad$ Cuida la pulcritud, ventilación y luminosidad del aula.

$\checkmark \quad$ Organiza comisiones de trabajo en el aula.

$\checkmark \quad$ Es organizado y cuidadoso con el material y documentos administrativos.

$\checkmark \quad$ Elabora y utiliza recursos.

8.2 Ambiente afectivo.

$\checkmark \quad$ Se preocupa por establecer sinergia con los alumnos.

$\checkmark \quad$ Establece un clima de sensibilidad para nuevos conocimientos.

$\checkmark \quad$ Promueve un clima seguro, cálido y confiable para el grupo.

$\checkmark \quad$ Toma en cuenta los planteamientos de los alumnos.

8.3 Ambiente para la convivencia.

$\checkmark \quad$ Considera la diversidad de los alumnos como un valor.

$\checkmark \quad$ Practica y motiva a practicar la tolerancia.

$\checkmark \quad$ Realiza actividades que ponen en juego la democracia.

$\checkmark \quad$ Promueve manifestaciones de trabajo en equipo y solidaridad.

$\checkmark \quad$ Ensalza el valor del trabajo productivo.

9. Autoaprendizaje.

9.1 Investigador.

$\checkmark \quad$ Manifiesta actitud de esmero y dedicación por la investigación. 
$\checkmark \quad$ Diagnóstica, programa, ejecuta y evalúa los procesos educativo.

$\checkmark \quad$ Pone en práctica el proceso de investigación-acción.

9.2 Evalúa el proceso de aprendizaje del alumno.

$\checkmark \quad$ Elabora y aplica instrumentos basados en las competencias e indicadores trabajados.

$\checkmark \quad$ Registro continuo de las evaluaciones. Sistematiza los aprendizajes.

$\checkmark \quad$ Atiende las características individuales de los alumnos.

9.3 Formación permanente.

$\checkmark \quad$ Aplica loas modalidades de investigación.

$\checkmark \quad$ Manejo de herramientas tecnológicas de aprendizaje.

$\checkmark \quad$ Uso de documentos bibliográficos.

$\checkmark \quad$ Sistematiza su práctica pedagógica.

$\checkmark \quad$ Aplica procesos metacognitivos.

$\checkmark \quad$ Planifica, concientiza, regula, supervisa y reorienta su práctica y aprendizaje.

10. Cualidades personales del docente.

10.1 Dominio del carácter.

$\checkmark \quad$ Controla sus emociones.

$\checkmark \quad$ Se pone en lugar del otro.

$\checkmark$ Toma decisiones acertadas.

$\checkmark \quad$ Tiene iniciativa.

$\checkmark \quad$ Colabora efectiva y espontáneamente.

$\checkmark \quad$ Amable y Tolerante.

10.2 Concepto de sí mismo.

$\checkmark \quad$ Confía en sí mismo.

$\checkmark \quad$ Valora sus logros.

$\checkmark \quad$ Se interesa por los cambios.

$\checkmark \quad$ Estudia e investiga causas.

$\checkmark \quad$ Establece relaciones adecuadas con los demás.

10.3 Actitudes.

$\checkmark \quad$ Se comunica con facilidad.

$\checkmark \quad$ Trabaja en equipo.

$\checkmark \quad$ Es organizado.

$\checkmark \quad$ Confía en el entorno institucional.

$\checkmark \quad$ Tiene sentido de pertenencia y pertinencia.

10.4 Valores.

$\checkmark \quad$ Puntual y Responsable.

$\checkmark \quad$ Respeta las reglas y normas.

$\checkmark \quad$ Asume compromisos y tareas.

$\checkmark \quad$ Es honesto y ético.

$\checkmark \quad$ Es tolerante, democrático y participativo. 


\section{CONCLUSIONES}

Las conclusiones fundamentales de la presente investigación son las siguientes:

1. Se identificaron los elementos esenciales de una competencia:

a) Características personales.

b) Que son causantes de acciones que producen resultados adecuados.

c) En diferentes contextos y situaciones.

d) Estos elementos funcionan como un sistema globalizado.

2. Se probó un método interactivo de producción de resultados de investigación con resultados positivos.

3. Se formuló una matriz del docente de educación básica con 10 competencias, subcompetencias e indicadores. Las diez competencias seleccionadas fueron: Motivación al logro, atención centrada en el alumno, sensibilidad social, equipo de aprendizaje, agente de cambio, dominio de contenidos básicos, dominio de estrategias de aprendizaje, Ambiente de aprendizaje adecuado, Autoaprendizaje, características personales.

\section{REFERENCIAS BIBLIOGRÁFICAS}

ARgüELles, Antonio. Competencia Laboral y Educación basada en Normas de Competencia. México: Editorial Limusa, Año 2000.

LEBOYER, Claude. Gestión de las Competencias. España: Ediciones Gestión 2000, Año 1997.

FAURE, Edgar. Aprender a Ser, UNESCO. Venezuela: Grupo Santillana de Ediciones, Año 1972.

Goleman, Daniel. La Inteligencia Emocional en al Empresa. Argentina: Ediciones B Argentina, Año 1999.

Goleman, Daniel. La Inteligencia Emocional. México: Ediciones B México, Año 1995.

HAYGROUP. Las Competencias: Clave para una gestión integrada de los Recursos Humanos. España: Ediciones Deusto, Año 1996.

UnIVERSIDAD DE ANTIOQUIA. Técnica de recolección de información mediante los grupos focales. Página Web en línea, disponible: http://huitoto.udea.edu.co/ ceo/Grupos_Focales.html, Año 2002.

PERRENOUD, Philippe. De nouvelles compétences professionnelles pour enseigner à l'école primaire. Página Web en línea, disponible: http://agora.unige.ch/ctie/educateur/perrint .htm, Año 1997.

Programa de las naciones Unidas Para El Desarrollo. Informe sobre el Desarrollo Humano. Página Web en línea, disponible: www.undp.org/hdr2001/spanish.htm, Año 2001.

UNESCO. ¿Qué es la UNESCO?. Página Web en línea, disponible: www.unesco.org, Año 2002.

UNESCO. Informe sobre la Educación en el Mundo 2000. Venezuela: Grupo Santillana de Ediciones, Año 2000.

UNESCO. Declaración Mundial sobre Educación para Todos. Documento en línea, disponible: http:// www.unesco.org, Año 2000.

UNESCO. Indicadores de Educación en el Mundo. Guinea: UNESCO, Año 1998.

DELORS, Jackes. La Educación Encierra un Tesoro. España: Grupo Santillana de Ediciones, Año 1996. 
VARGAS, Fernándo. 40 Preguntas sobre Competencia Laboral. Uruguay: Cinterfor, Año 2004

VARGAS, Fernándo. Clasificaciones de ocupaciones, competencias y formación profesional: ¿Paralelismo o Convergencia? Documento en línea, disponible: http://www.ilo.org/public/spanish/region/ampro/cinterfor/publ/ sala/vargas/clasific/index.htm.2002 


\title{
Contactar
}

Revista lberoamericana de Educación

\author{
Principal OEI
}

